\title{
Exclusive diffractive photon bremsstrahlung at the LHC
}

\author{
Piotr Lebiedowicz ${ }^{1, *}$ and Antoni Szczurek ${ }^{1,2, \oplus}$ \\ ${ }^{1}$ Institute of Nuclear Physics PAN, PL-31-342 Cracow, Poland \\ ${ }^{2}$ University of Rzeszów, PL-35-959 Rzeszów, Poland
}

\begin{abstract}
We calculate differential distributions for the $p p \rightarrow p p \gamma$ reaction at the LHC energy $\sqrt{s}=14 \mathrm{TeV}$. We consider diffractive classical bremsstrahlung mechanisms including effects of non point-like nature of protons. In addition, we take into account (vector meson)-pomeron, photon-pion as well as photon-pomeron exchange processes for the first time in the literature. Predictions for the total cross section and several observables related to these processes e.g. differential distributions in pseudorapidities and transverse momenta of photons or protons are shown and discussed. The integrated diffractive bremsstrahlung cross section $\left(E_{\gamma}>100 \mathrm{GeV}\right)$ is only of the order of $\mu$ b. We try to identify regions of the phase space where one of the mechanisms dominates. The classical bremsstrahlung dominates at large forward/backward photon pseudorapidities, close to the pseudorapidities of scattered protons. In contrast, the photon-pomeron (pomeron-photon) mechanism dominates at midrapidities but the related cross section is rather small. In comparison the virtualomega rescattering mechanism contributes at smaller angles of photons (larger photon rapidities). Photons in the forward/backward region can be measured by the Zero Degree Calorimeters (ZDCs) installed in experiments at the LHC while the midrapidity photons are difficult to measure (small cross section, small photon transverse momenta). Protons could be measured by ALFA detector (ATLAS) or TOTEM detector at CMS. The exclusivity could be checked with the help of main central detectors.
\end{abstract}

PACS numbers: 13.60.Le, 13.85.-t, 14.40.Be, 12.40.Nn

* Piotr.Lebiedowicz@ifj.edu.pl

$\dagger$ Antoni.Szczurek@ifj.edu.pl 


\section{INTRODUCTION}

Exclusive diffractive photon bremsstrahlung mechanism at high energies was almost not studied in the literature. Because at high energy the pomeron exchange is the driving mechanism of bremsstrahlung it is logical to call the mechanisms described by the diagrams shown in Fig.1 diffractive bremsstrahlung to distinguish from the low-energy bremsstrahlung driven by meson exchanges 1 . The exclusive photon production mechanism is similar to $p p \rightarrow p p \omega$ [3] and $p p \rightarrow p p \pi^{0}$ [4] processes. As discussed in the past the dominant hadronic bremsstrahlung-type mechanism is the Drell-Hiida-Deck (DHD) mechanism [5] for diffractive production of $\pi N$ final states (for a nice review we refer to [6] and references therein).

The $p p \rightarrow p p \gamma$ process at high energies was discussed only recently [7] and it was proposed to use the exclusive photon bremsstrahlung to measure or estimate elastic proton-proton cross section at the LHC. Only approximate formulas for the classical bremsstrahlung were given there. The participating particles were treated there as point-like particles. No differential distributions for the exclusive bremsstrahlung have been discussed.

The photons radiated off the initial and final state protons can be seen by the Zero Degree Calorimeters (ZDCs) that are installed at about 140 meters on each side of the interaction region. They will measure very forward neutral particles in the pseudorapidity region $|\eta|>$ 8.5 at the CMS [8] and the ATLAS ZDCs provide coverage of the region $|\eta|>8.3$ [9] 2 . The forward detectors beyond pseudorapidities of $|\eta|>3$ provide an efficient veto against neutral particle backgrounds in the ZDCs from diffractive and non-diffractive events (see [7]). Furthermore the proposed Forward Shower Counters (FSCs), to detect and trigger on rapidity gaps in diffractive events, would improve the measurements at the LHC significantly 11]. In addition to a measurement of the elastic $p p$ cross section the bremsstrahlung photons could allow for the evaluation of the total $p p$ cross section, luminosity and relative alignment of the ZDCs and of the Roman Pot detectors.

In this paper, we wish to present a first detailed studies of single photon bremsstrahlung in the exclusive process $p p \rightarrow p p \gamma$. We shall include classical bremsstrahlung diagrams as well as some new diagrams characteristic exclusively for proton-proton scattering, not present e.g. in $e^{+} e^{-}$scattering. We include diagrams which arise in the vector-dominance model as well as photon-pion (pion-photon) and photon-pomeron (pomeron-photon) exchange processes not discussed so far in the literature. We shall try to identify the region of the phase space where one can expect a dominance of one of the processes through detailed studies of several differential distributions.

\section{THE AMPLITUDES FOR THE $p p \rightarrow p p \gamma$ REACTION}

\section{A. Diffractive classical bremsstrahlung mechanisms}

The bremsstrahlung mechanisms for exclusive production of photons discussed here are shown schematically in Fig.1. In the case of $\gamma$ production the diagrams with intermediate

${ }^{1}$ The photon bremsstrahlung was intensively studied in nucleon-nucleon collisions at low energies (see e.g. [1, 2] and references therein). There the dominant mechanisms are nucleon current (off-shell nucleon) and/or mesonic current (photon emitted from the middle of exchanged mesons) contributions driven by meson exchanges.

${ }^{2}$ Recently, the exclusive $p p \rightarrow n n \pi^{+} \pi^{+}$reaction has been studied in Ref.[10]. This reaction can be also measured with the help of the ZDC detectors. Very large cross sections has been found which is partially due to interference of a few mechanisms. Presence of several interfering mechanisms precludes extraction of the elastic $\pi^{+} \pi^{+}$scattering cross section. 
a)

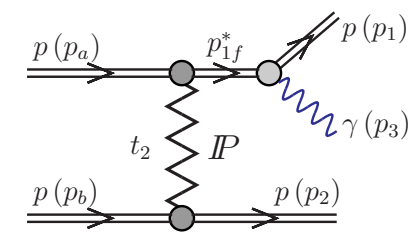

b)

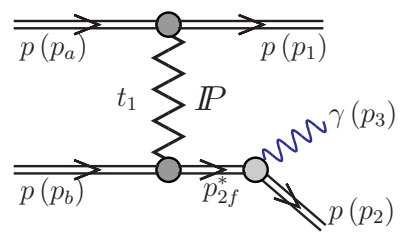

c)

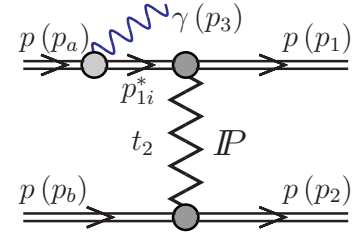

d)

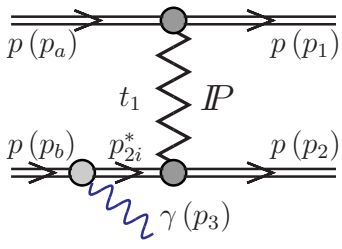

FIG. 1. Diagrams of the bremsstrahlung amplitudes driven by the pomeron exchange.

nucleon resonances (see [13]) should be negligible. The pronounced at low energy proton to $\Delta$ isobar transitions are suppressed in high energy regime.

The Born amplitudes of diagrams shown in Fig 1 can be written as

$$
\begin{aligned}
\mathcal{M}_{\lambda_{a} \lambda_{b} \rightarrow \lambda_{1} \lambda_{2} \lambda_{3}}^{(a)} & =e \bar{u}\left(p_{1}, \lambda_{1}\right) \ddagger^{*}\left(p_{3}, \lambda_{3}\right) S_{N}\left(p_{1 f}^{2}\right) \gamma^{\mu} u\left(p_{a}, \lambda_{a}\right) F_{\gamma N^{*} N}\left(p_{1 f}^{2}\right) F_{\mathbb{P} N N^{*}}\left(p_{1 f}^{2}\right) \\
& \times i s C_{\mathbb{P}}^{N N}\left(\frac{s}{s_{0}}\right)^{\alpha_{\mathbb{P}}\left(t_{2}\right)-1} \exp \left(\frac{B_{\mathbb{P}}^{N N} t_{2}}{2}\right) \frac{1}{2 s} \bar{u}\left(p_{2}, \lambda_{2}\right) \gamma_{\mu} u\left(p_{b}, \lambda_{b}\right) \\
\mathcal{M}_{\lambda_{a} \lambda_{b} \rightarrow \lambda_{1} \lambda_{2} \lambda_{3}}^{(b)} & =e \bar{u}\left(p_{2}, \lambda_{2}\right) \ddagger^{*}\left(p_{3}, \lambda_{3}\right) S_{N}\left(p_{2 f}^{2}\right) \gamma^{\mu} u\left(p_{b}, \lambda_{b}\right) F_{\gamma N^{*} N}\left(p_{2 f}^{2}\right) F_{\mathbb{P} N N^{*}}\left(p_{2 f}^{2}\right) \\
& \times i s C_{\mathbb{P}}^{N N}\left(\frac{s}{s_{0}}\right)^{\alpha_{\mathbb{P}}\left(t_{1}\right)-1} \exp \left(\frac{B_{\mathbb{P}}^{N N} t_{1}}{2}\right) \frac{1}{2 s} \bar{u}\left(p_{1}, \lambda_{1}\right) \gamma_{\mu} u\left(p_{a}, \lambda_{a}\right) \\
& \times i s_{12} C_{\mathbb{P}}^{N N}\left(\frac{s_{12}}{s_{0}}\right)^{\alpha_{\mathbb{P}}\left(t_{2}\right)-1} \exp \left(\frac{B_{\mathbb{P}}^{N N} t_{2}}{2}\right) \frac{1}{2 s_{12}} \bar{u}\left(p_{2}, \lambda_{2}\right) \gamma_{\mu} u\left(p_{b}, \lambda_{b}\right), \\
\mathcal{M}_{\lambda_{a} \lambda_{b} \rightarrow \lambda_{1} \lambda_{2} \lambda_{3}}^{(c)} & =e \bar{u}\left(p_{1}, \lambda_{1}\right) \gamma^{\mu} S_{N}\left(p_{1 i}^{2}\right) \ddagger^{*}\left(p_{3}, \lambda_{3}\right) u\left(p_{a}, \lambda_{a}\right) F_{\gamma N N^{*}}\left(p_{1 i}^{2}\right) F_{\mathbb{P} N^{*} N}\left(p_{1 i}^{2}\right) \\
\mathcal{M}_{\lambda_{a} \lambda_{b} \rightarrow \lambda_{1} \lambda_{2} \lambda_{3}}^{(d)} & =e \bar{u}\left(p_{2}, \lambda_{2}\right) \gamma^{\mu} S_{N}\left(p_{2 i}^{2}\right) \ddagger^{*}\left(p_{3}, \lambda_{3}\right) u\left(p_{b}, \lambda_{b}\right) F_{\gamma N N^{*}}\left(p_{2 i}^{2}\right) F_{\mathbb{P} N^{*} N}\left(p_{2 i}^{2}\right) \\
& \times i s_{12} C_{\mathbb{P}}^{N N}\left(\frac{s_{12}}{s_{0}}\right)^{\alpha_{\mathbb{P}}\left(t_{1}\right)-1} \exp \left(\frac{B_{\mathbb{P}}^{N N} t_{1}}{2}\right) \frac{1}{2 s_{12}} \bar{u}\left(p_{1}, \lambda_{1}\right) \gamma_{\mu} u\left(p_{a}, \lambda_{a}\right),
\end{aligned}
$$

where $u(p, \lambda), \bar{u}\left(p^{\prime}, \lambda^{\prime}\right)=u^{\dagger}\left(p^{\prime}, \lambda^{\prime}\right) \gamma^{0}$ are the Dirac spinors (normalized as $\bar{u}\left(p^{\prime}\right) u(p)=2 m_{p}$ ) of the initial and outgoing protons with the four-momentum $p$ and the helicities $\lambda$. The factor $\frac{1}{2 s}$ or $\frac{1}{2 s_{12}}$ appear here as a consequence of using spinors. The four-momenta squared of virtual nucleons in the middle of diagrams are defined as $t_{1,2}=q_{1,2}^{2}=\left(p_{a, b}-p_{1,2}\right)^{2}$, $p_{1 i, 2 i}^{2}=\left(p_{a, b}-p_{3}\right)^{2}, p_{1 f, 2 f}^{2}=\left(p_{1,2}+p_{3}\right)^{2}$ and $s_{i j}=W_{i j}^{2}=\left(p_{i}+p_{j}\right)^{2}$ are squared invariant masses of the $(i, j)$ system. The propagators of the intermediate nucleons can be written as

$$
S_{N}\left(p^{2}\right)=\frac{i\left(p+m_{p}\right)}{p^{2}-m_{p}^{2}},
$$

where $\not p=p_{\mu} \gamma^{\mu}$. The polarization vectors of real photon $\left(\AA^{*}\left(p_{3}, \lambda_{3}\right)=\gamma^{\nu} \varepsilon_{\nu}^{*}\left(p_{3}, \lambda_{3}\right)\right)$ are defined in the proton-proton center-of-mass frame

$$
\varepsilon_{\nu}\left(p_{3}, \pm 1\right)=\frac{1}{\sqrt{2}}(0, i \sin \phi \mp \cos \theta \cos \phi,-i \cos \phi \mp \cos \theta \sin \phi, \pm \sin \theta)
$$


where $\theta$ is the polar angle and $\phi$ is the azimuthal angle of a emitted photon. It is easy to check that they fulfill the relation $\varepsilon^{\nu}(p, \lambda) \varepsilon_{\nu}^{*}(p, \lambda)=-1$ and $p^{\nu} \varepsilon_{\nu}(p, \lambda)=0$.

We use interaction parameters of Donnachie-Landshoff [14] with $C_{\mathbb{P}}^{N N}=21.7 \mathrm{mb}$ and the pomeron trajectory $\alpha_{\mathbb{P}}(t)$ linear in $t$

$$
\alpha_{\mathbb{P}}(t)=\alpha_{\mathbb{P}}(0)+\alpha_{\mathbb{P}}^{\prime} t, \quad \alpha_{\mathbb{P}}(0)=1.0808, \quad \alpha_{\mathbb{P}}^{\prime}=0.25 \mathrm{GeV}^{-2} .
$$

The pomeron slope can be written as

$$
B(s)=B_{\mathbb{I P}}^{N N}+2 \alpha_{\mathbb{P}}^{\prime} \ln \left(\frac{s}{s_{0}}\right)
$$

where we use $s_{0}=1 \mathrm{GeV}^{2}$ and $B_{\mathbb{P}}^{N N}=9 \mathrm{GeV}^{-2}$ which approximately describes a running slope for proton-proton elastic scattering. Since in our calculations we include effective pomerons, i.e. pomerons describing approximately nucleon-nucleon elastic scattering, no explicit absorption corrections have to be included in addition.

In the bremsstrahlung processes discussed here the intermediate nucleons are off-mass shell. In our approach the off-shell effects related to the non-point-like protons in the intermediate state are included by the following simple extra form factors

$$
F\left(p^{2}\right)=\frac{\Lambda_{N}^{4}}{\left(p^{2}-m_{p}^{2}\right)^{2}+\Lambda_{N}^{4}} .
$$

This form was used e.g. in Ref.[12] for $\omega$ photoproduction. In general, the cut-off parameters in the form factors are not known but could be fitted in the future to the (normalized) experimental data. From our general experience in hadronic physics we expect $\Lambda_{N} \sim 1 \mathrm{GeV}$. We shall discuss how the uncertainties of the form factors influence our final results.

We could "improve" the parametrization of the amplitudes (2.3) and (2.4) to reproduce the high-energy Regge dependence by the factors $\left(s_{13} / m_{p}^{2}\right)^{\alpha_{N}\left(p_{1 i}^{2}\right)-\frac{1}{2}}$ and $\left(s_{23} / m_{p}^{2}\right)^{\alpha_{N}\left(p_{2 i}^{2}\right)-\frac{1}{2}}$, respectively, where the nucleon trajectory is $\alpha_{N}\left(p_{1 i, 2 i}^{2}\right)=-0.3+\alpha_{N}^{\prime} p_{1 i, 2 i}^{2}$, with $\alpha_{N}^{\prime}=0.9$ $\mathrm{GeV}^{-2}$. We leave the problem of consistent nucleon reggezation in the context of high-energy photon bremsstrahlung for future studies.

\section{B. Bremsstrahlung of $\omega$ mesons}

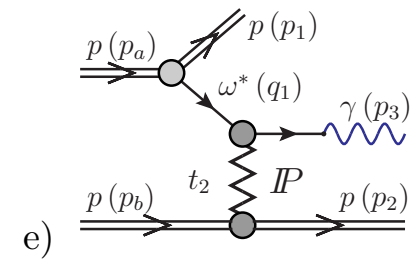

f)

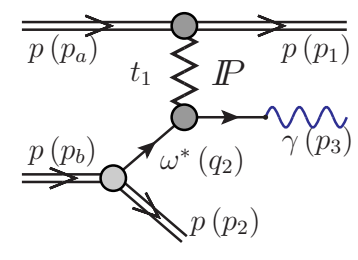

FIG. 2. Diagrams of the bremsstrahlung amplitudes with virtual- $\omega$ meson (reggeon) and its transformation to final state photon.

In Ref. [3] we have discussed bremsstrahlung of $\omega$ mesons. There one includes processes when $\omega$ meson emitted by an (anti)proton interacts with the second (anti)proton. The Born 
amplitudes for the interaction with emitted virtual- $\omega$ meson and its subsequent transformation to a photon, shown in Fig, 2, are obtained as

$$
\begin{aligned}
\mathcal{M}_{\lambda_{a} \lambda_{b} \rightarrow \lambda_{1} \lambda_{2} \lambda_{3}}^{(e) \omega \mathbb{P}-\text { exch. }} & =\bar{u}\left(p_{1}, \lambda_{1}\right) \gamma^{\mu} u\left(p_{a}, \lambda_{a}\right) S_{\mu \nu}\left(t_{1}\right) \varepsilon^{\nu *}\left(p_{3}, \lambda_{3}\right) g_{\omega N N} F_{\omega^{*} N N}\left(t_{1}\right) F_{\mathbb{P} \omega^{*} \omega}\left(t_{1}\right) C_{\omega \rightarrow \gamma} \\
& \times i s_{23} C_{\mathbb{P}}^{\omega N}\left(\frac{s_{23}}{s_{0}}\right)^{\alpha_{\mathbb{P}}\left(t_{2}\right)-1}\left(\frac{s_{13}}{s_{t h r}}\right)^{\alpha_{\omega}\left(t_{1}\right)-1} \exp \left(\frac{B_{\mathbb{P}}^{\omega N} t_{2}}{2}\right) \delta_{\lambda_{2} \lambda_{b}}, \\
\mathcal{M}_{\lambda_{a} \lambda_{b} \rightarrow \lambda_{1} \lambda_{2} \lambda_{3}}^{(f) \mathbb{P} \omega-e x c h .} & =\bar{u}\left(p_{2}, \lambda_{2}\right) \gamma^{\mu} u\left(p_{b}, \lambda_{b}\right) S_{\mu \nu}\left(t_{2}\right) \varepsilon^{\nu *}\left(p_{3}, \lambda_{3}\right) g_{\omega N N} F_{\omega^{*} N N}\left(t_{2}\right) F_{\mathbb{P} \omega^{*} \omega}\left(t_{2}\right) C_{\omega \rightarrow \gamma} \\
& \times i s_{13} C_{\mathbb{P}}^{\omega N}\left(\frac{s_{13}}{s_{0}}\right)^{\alpha_{\mathbb{P}}\left(t_{1}\right)-1}\left(\frac{s_{23}}{s_{t h r}}\right)^{\alpha_{\omega}\left(t_{2}\right)-1} \exp \left(\frac{B_{\mathbb{P}}^{\omega N} t_{1}}{2}\right) \delta_{\lambda_{1} \lambda_{a}},
\end{aligned}
$$

where $S_{\mu \nu}(t)$ is the propagator of the $\omega$ meson

$$
S_{\mu \nu}(t)=\frac{-g_{\mu \nu}+\frac{q_{\mu} q_{\nu}}{m_{\omega}^{2}}}{t-m_{\omega}^{2}}
$$

In our calculation we assume $C_{\mathbb{P}}^{\omega N}=C_{\mathbb{P}}^{\pi N}=13.63 \mathrm{mb}$ [14] and the slope parameter $B_{\mathbb{P}}^{\omega N}=B_{\mathbb{P}}^{\pi N}=5.5 \mathrm{GeV}^{-2}$ (see e.g. [3, 16]). The amplitudes above, (2.10) and (2.11), are corrected to reproduce the high-energy Regge dependence by the Regge-like factors $\left(s_{13} / s_{t h r}\right)^{\alpha_{\omega}\left(t_{1}\right)-1}$ and $\left(s_{23} / s_{t h r}\right)^{\alpha_{\omega}\left(t_{2}\right)-1}$, respectively. The $\omega$-reggeon trajectory is taken as $\alpha_{\omega}(t)=0.5+0.9 t$ and $s_{t h r}=\left(m_{p}+m_{\omega}\right)^{2}$.

Different values of the omega meson to nucleon coupling constant have been used in the literature [17]. In our calculation we assume coupling constant $g_{\omega N N}^{2} / 4 \pi=10$. Similar value was used in [18, 19]. The transformation of $\omega$ meson to photon is obtained within vector dominance model [20] and $C_{\omega \rightarrow \gamma}=\sqrt{\alpha_{e m} / 20.5} \simeq 0.02, \alpha_{e m}=e^{2} /(4 \pi)$. For completeness of this analysis we should include also amplitudes for the interaction with emitted (virtual) $\rho$ meson. Because of isospin, there is no mixing between the intermediate $\omega$ and $\rho$ mesons. The transition of $\rho$ meson to photon is more probable $C_{\rho \rightarrow \gamma}=\sqrt{\alpha_{e m} / 2.54} \simeq 0.05$ whereas the coupling constant $g_{\rho N N}$ is small compared with $g_{\omega N N}$ - in consequence the $\rho-\mathbb{P}$ contribution is comparable to the $\omega-\mathbb{P}$ contribution.

The off-shell form factors $F_{\omega^{*} N N}$ and $F_{\mathbb{I P} \omega^{*} \omega}$ in (2.10) and (2.11) will be taken here in the following exponential form:

$$
F_{\omega^{*} N N}(t)=\exp \left(\frac{t-m_{\omega}^{2}}{\Lambda_{\omega N N}^{2}}\right), \quad F_{\mathbb{I P} \omega^{*} \omega}(t)=\exp \left(\frac{t-m_{\omega}^{2}}{\Lambda_{\mathbb{P} \omega \omega}^{2}}\right)
$$

where $\Lambda_{\omega N N}=\Lambda_{\mathbb{P} \omega \omega}=1 \mathrm{GeV}$.

\section{Pion cloud and $\gamma \pi^{0}$ and $\pi^{0} \gamma$ exchanges}

In our present analysis we include also $\gamma \pi^{0}$ and $\pi^{0} \gamma$ exchanges. The underlying mechanisms are shown in Fig.3. Such diagrams are dictated by the presence of pion cloud in the nucleon (see e.g. [21]). 


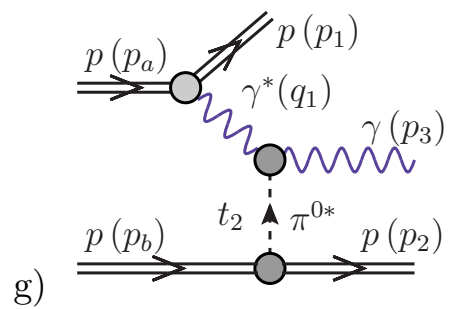

h)

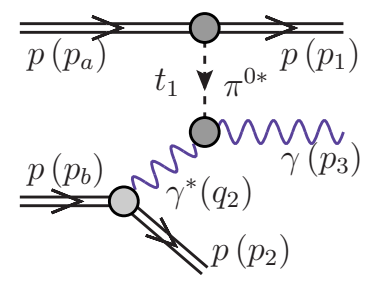

FIG. 3. Diagrams with the $\gamma \pi^{0}$ and $\pi^{0} \gamma$ exchanges in the $p p \rightarrow p p \gamma$ reaction.

The amplitudes for the two new processes can be easily written as:

$$
\begin{aligned}
\mathcal{M}_{\lambda_{a} \lambda_{b} \rightarrow \lambda_{1} \lambda_{2} \lambda_{3}}^{(g) \gamma \pi^{0}-e x c h} & =e \bar{u}\left(p_{1}, \lambda_{1}\right) \gamma^{\alpha} u\left(p_{a}, \lambda_{a}\right) F_{1}\left(t_{1}\right) \\
& \times \frac{-g_{\alpha \beta}}{t_{1}} F_{\gamma \pi \rightarrow \gamma}\left(t_{1}, t_{2}\right) \varepsilon^{\beta \mu \nu \lambda} q_{1 \mu} p_{3 \nu} \varepsilon_{\lambda}^{*}\left(p_{3}, \lambda_{3}\right) \\
& \times g_{\pi^{0} N N} F_{\pi N N}\left(t_{2}\right) \frac{1}{t_{2}-m_{\pi}^{2}} \bar{u}\left(p_{2}, \lambda_{2}\right) i \gamma_{5} u\left(p_{b}, \lambda_{b}\right) \\
\mathcal{M}_{\lambda_{a} \lambda_{b} \rightarrow \lambda_{1} \lambda_{2} \lambda_{3}}^{(h) \pi^{0} \gamma-e x c h .} & =g_{\pi^{0} N N} F_{\pi N N}\left(t_{1}\right) \frac{1}{t_{1}-m_{\pi}^{2}} \bar{u}\left(p_{1}, \lambda_{1}\right) i \gamma_{5} u\left(p_{a}, \lambda_{a}\right) \\
& \times \frac{-g_{\alpha \beta}}{t_{2}} F_{\gamma \pi \rightarrow \gamma}\left(t_{2}, t_{1}\right) \varepsilon^{\beta \mu \nu \lambda} q_{2 \mu} p_{3 \nu} \varepsilon_{\lambda}^{*}\left(p_{3}, \lambda_{3}\right) \\
& \times e \bar{u}\left(p_{2}, \lambda_{2}\right) \gamma^{\alpha} u\left(p_{b}, \lambda_{b}\right) F_{1}\left(t_{2}\right),
\end{aligned}
$$

where the $\gamma^{*} N N$-vertices are parametrized by the proton Dirac electromagnetic form factors

$$
F_{1}(t)=\frac{4 m_{p}^{2}-2.79 t}{\left(4 m_{p}^{2}-t\right)\left(1-t / m_{D}^{2}\right)^{2}},
$$

where $m_{p}$ is the proton mass and $m_{D}^{2}=0.71 \mathrm{GeV}^{2}$. The coupling of the pion to the nucleon $g_{\pi N N}^{2} / 4 \pi=13.5$ is relatively well known (see e.g. [22]) and the corresponding hadronic form factor is taken in the exponential form

$$
F_{\pi N N}(t)=\exp \left(\frac{t-m_{\pi}^{2}}{\Lambda_{\pi N N}^{2}}\right)
$$

with $\Lambda_{\pi N N}=1 \mathrm{GeV}$. For the central vertices involving off-shell particles the $\gamma \pi^{0}$ form factors are taken in the following factorized form

$$
F_{\gamma \pi \rightarrow \gamma}\left(t_{1}, t_{2}\right)=\frac{N_{c}}{12 \pi^{2} f_{\pi}} \frac{m_{\rho}^{2}}{m_{\rho}^{2}-t_{1}} \exp \left(\frac{t_{2}-m_{\pi}^{2}}{\Lambda_{\gamma \pi \rightarrow \gamma}^{2}}\right)
$$

with the pion decay constant $f_{\pi}=93 \mathrm{MeV}$ and $N_{c}=3$. The factor describing the virtual photon coupling is taken as in the vector dominance model. In practical calculations we take $\Lambda_{\gamma \pi \rightarrow \gamma}=1 \mathrm{GeV}$.

\section{Photon rescattering, $\gamma \mathbb{P}$ and $\mathbb{P} \gamma$ exchanges}

At high energy there is still another type of diagrams (mechanisms) shown in Fig.4. We shall call them in the following diagrams i) and j) for simplicity. Here the intermediate 

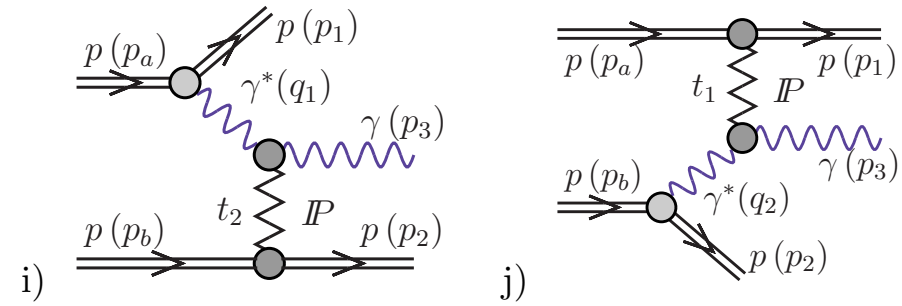

FIG. 4. Diagrams with the $\gamma \mathbb{P}$ and $\mathbb{P} \gamma$ exchanges for the $p p \rightarrow p p \gamma$ reaction.

photon couples to one of the protons through electromagnetic form factors and interacts (at high energies) with the second proton exchanging pomeron (phenomenology) or gluonic ladder in the QCD language (see e.g. [15]). This is a counterpart of the diagrams g) and h) (see Fig 3) relevant at lower $\gamma p$ subenergies.

The amplitude of the three-body process can be written in terms of the amplitude for elastic $\gamma p$ scattering. For not too large $t$ the $\gamma p \rightarrow \gamma p$ amplitude can be simply parametrized as

$$
\mathcal{M}_{\gamma p \rightarrow \gamma p}(s, t) \cong i s \sigma_{\text {tot }}^{\gamma p}(s) \exp \left(\frac{B_{\gamma p}}{2} t\right)
$$

Such an amplitude gives, however, correct total cross section by construction. In the calculations presented in the Result section we shall use the simple Donnachie-Landshoff fit to the world data on photon-proton total cross section [14] in which the pomeron and subleading reggeon exchanges 3 have been included

$$
\begin{aligned}
& \sigma_{\text {tot }}^{\gamma p}(s)=C_{\mathbb{P}}^{\gamma p} s^{\alpha_{\mathbb{P}}(0)-1}+C_{\mathbb{R}}^{\gamma p} s^{\alpha_{\mathbb{R}}(0)-1}, \\
& C_{\mathbb{P}}^{\gamma p}=0.0677 \mathrm{mb}, \quad C_{\mathbb{R}}^{\gamma p}=0.129 \mathrm{mb}, \quad \alpha_{\mathbb{P}}(0)=1.0808, \quad \alpha_{\mathbb{R}}(0)=0.5475 .
\end{aligned}
$$

In general, the slope parameter could be found by fitting to elastic $\gamma p$ scattering data which are, however, unknown and very difficult to measure. Since the incoming photon must first fluctuate to the $q \bar{q}$ state which interacts by the pomeron exchange with a proton before forming the outgoing vector meson, it seems reasonable to use a hadronic slope for a first estimation. In practical calculations we shall use $B_{\gamma p}(s)=B_{\pi p}(s)$ with its energy dependence, see Eq.(2.8).

Having fixed the elementary $\gamma p \rightarrow \gamma p$ amplitude we can proceed to our three-body photon rescattering amplitude. Limiting to large energies and small transverse momenta $t_{1}$ and $t_{2}$, helicity conserving processes, the matrix element can be written as

$$
\begin{aligned}
\mathcal{M}_{\lambda_{a} \lambda_{b} \rightarrow \lambda_{1} \lambda_{2} \lambda_{3}} \cong \delta_{\lambda_{2} \lambda_{b}} \mathcal{M}_{\gamma p \rightarrow \gamma p}\left(s_{23}, t_{2}\right) \frac{e F_{1}\left(t_{1}\right)}{t_{1}}\left(p_{a}+p_{1}\right)^{\mu} \varepsilon_{\mu}^{*}\left(p_{3}, \lambda_{3}\right) \delta_{\lambda_{1} \lambda_{a}} \\
+\delta_{\lambda_{1} \lambda_{a}} \mathcal{M}_{\gamma p \rightarrow \gamma p}\left(s_{13}, t_{1}\right) \frac{e F_{1}\left(t_{2}\right)}{t_{2}}\left(p_{b}+p_{2}\right)^{\mu} \varepsilon_{\mu}^{*}\left(p_{3}, \lambda_{3}\right) \delta_{\lambda_{2} \lambda_{b}} .
\end{aligned}
$$

Using $\vec{q}_{1,2 \perp}=-\vec{p}_{1,2 \perp}$ we have then

$$
\begin{aligned}
\mathcal{M}_{\lambda_{a} \lambda_{b} \rightarrow \lambda_{1} \lambda_{2} \lambda_{3}} \cong \delta_{\lambda_{2} \lambda_{b}} \mathcal{M}_{\gamma p \rightarrow \gamma p}\left(s_{23}, t_{2}\right) \frac{e F_{1}\left(t_{1}\right)}{t_{1}} \frac{2}{z_{1}} \frac{V^{*}\left(q_{1 \perp}, \lambda_{3}\right)}{\sqrt{1-z_{1}}} \delta_{\lambda_{1} \lambda_{a}} \\
+\delta_{\lambda_{1} \lambda_{a}} \mathcal{M}_{\gamma p \rightarrow \gamma p}\left(s_{13}, t_{1}\right) \frac{e F_{1}\left(t_{2}\right)}{t_{2}} \frac{2}{z_{2}} \frac{V^{*}\left(q_{2 \perp}, \lambda_{3}\right)}{\sqrt{1-z_{2}}} \delta_{\lambda_{2} \lambda_{b}},
\end{aligned}
$$

\footnotetext{
${ }^{3}$ In the reggeon contribution the $f_{2}$ exchange dominates over the $a_{2}$ exchange similarly as in the hadronic reactions, see [23].
} 
where the longitudinal momentum fractions of outgoing protons $z_{1,2}$ are

$$
z_{1} \cong \frac{s_{23}}{s}, \quad z_{2} \cong \frac{s_{13}}{s}, \quad z_{1}, z_{2}<1
$$

and $V\left(q_{\perp}, \lambda_{3}\right)$ can be calculated from $x, y$-components of momenta of participating protons

$$
V\left(q_{\perp}, \lambda_{3}= \pm 1\right)=\mathbf{e}_{\mu}^{\left(\lambda_{3}\right)} q_{\perp}^{\mu}=-\frac{1}{\sqrt{2}}\left(\lambda_{3} q_{x}+i q_{y}\right)
$$

\section{E. Equivalent Photon Approximation}

The cross section for $\gamma \pi^{0}$ exchange and $\gamma \mathbb{P}$ exchange mechanisms can be also calculated in the Equivalent Photon Approximation (EPA). In this approach the distribution of the photon can be written as

$$
\begin{aligned}
\frac{d \sigma}{d y d p_{\perp}^{2}} & =z_{1} f\left(z_{1}\right) \frac{d \sigma_{\gamma p \rightarrow \gamma p}}{d t_{2}}\left(s_{23}, t_{2} \approx-p_{\perp}^{2}\right) \\
& +z_{2} f\left(z_{2}\right) \frac{d \sigma_{\gamma p \rightarrow \gamma p}}{d t_{1}}\left(s_{13}, t_{1} \approx-p_{\perp}^{2}\right)
\end{aligned}
$$

where $f(z)$ is a photon flux in the proton; an explicit formula can be found e.g. in [24]. The differential distribution of elastic scattering at high energies is parametrized as

$$
\frac{d \sigma_{\gamma p \rightarrow \gamma p}}{d t}(s, t)=\frac{\left|\mathcal{M}_{\gamma p \rightarrow \gamma p}(s, t)\right|^{2}}{16 \pi s^{2}} .
$$

First energy and the longitudinal momentum of photon is calculated as a function of photon rapidity and transverse momentum $p_{\perp}=\sqrt{p_{x}^{2}+p_{y}^{2}}$

$$
E_{\gamma}=p_{\perp} \cosh y, \quad p_{z}=p_{\perp} \sinh y .
$$

We get

$$
p_{z}=\sqrt{E_{\gamma}^{2}-p_{\perp}^{2}} \text { for } y>0, \text { and } p_{z}=-\sqrt{E_{\gamma}^{2}-p_{\perp}^{2}} \text { for } y<0 .
$$

Then energies in the photon-proton subsystems can be calculated approximately as

$$
\begin{aligned}
& s_{13} \approx\left(p_{a 0}+E_{\gamma}\right)^{2}-\left(p_{a z}+p_{z}\right)^{2}, \\
& s_{23} \approx\left(p_{b 0}+E_{\gamma}\right)^{2}-\left(p_{b z}+p_{z}\right)^{2} .
\end{aligned}
$$

The fractional energy, $z_{1}$ and $z_{2}$, losses of the protons with four-momenta $p_{a}$ and $p_{b}$, respectively, can be obtained from Eq.(2.23).

\section{RESULTS}

In the following section we shall show results of the differential distributions for the exclusive bremsstrahlung mechanisms discussed in the previous section. The amplitudes for processes discussed in the sections above are calculated numerically for each point in the phase space. In calculating cross section of the three-body process we perform integrations in $\xi_{1}=\log _{10}\left(p_{1 \perp} / 1 \mathrm{GeV}\right)$ and $\xi_{2}=\log _{10}\left(p_{2 \perp} / 1 \mathrm{GeV}\right)$ instead in $p_{1 \perp}$ and $p_{2 \perp}$, in the photon 
(pseudo)rapidity $\eta_{\gamma}$ and the relative azimuthal angle between the outgoing protons $\phi_{12}=$ $\phi_{1}-\phi_{2}$.

The photon energy spectrum drops relatively slowly with photon energy as is shown in Fig 5 (left panel). The ZDC detectors (at ATLAS or CMS) can measure only photons above some energy threshold (e.g. $E_{\gamma}>50 \mathrm{GeV}$ ). In the calculation of classical bremsstrahlung presented here we assume $E_{\gamma}>100 \mathrm{GeV}$ as an example. Corresponding distributions in the photon transverse momentum are shown in Fig.5 (right panel). The contribution of classical bremsstrahlung is concentrated at very small transverse momenta which is consistent with very small photon emission angle (large pseudorapidity). The other distributions have rather similar shape and vanish at $p_{\perp, \gamma}=0 \mathrm{GeV}$. The exact shape may depend somewhat on the functional form and values of cut-off parameters of off-shell form factors taking into account the non-point-like nature of the vertices involved. Here we have fixed the values of the corresponding form factors at typical hadronic scales.
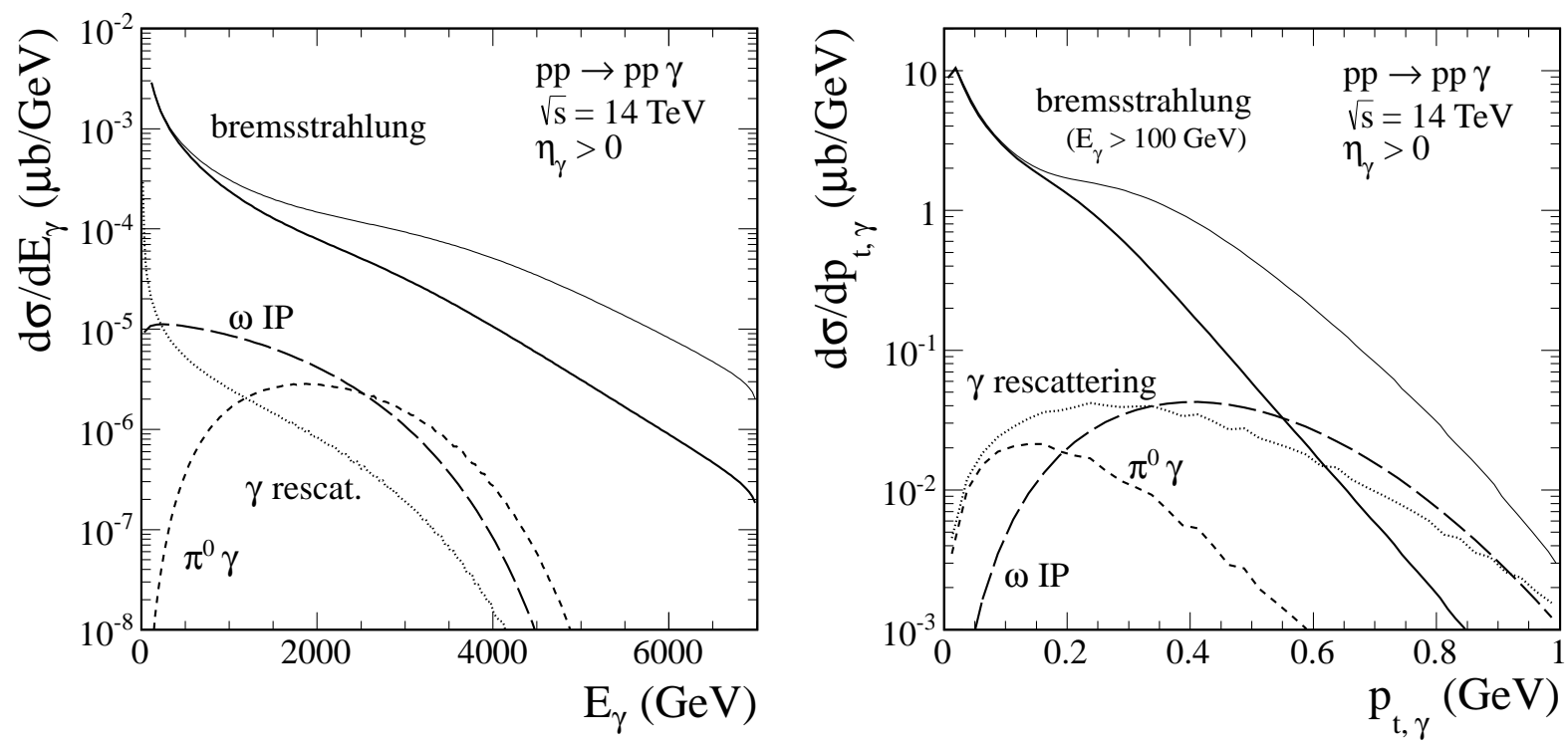

FIG. 5. Energy spectrum of photons (left panel) and distribution in transverse momentum of photons (right panel) for all processes considered here at $\sqrt{s}=14 \mathrm{TeV}$ and $\eta_{\gamma}>0$. For classical bremsstrahlung we have imposed $E_{\gamma}>100 \mathrm{GeV}$ and used two values $\Lambda_{N}=0.8,1 \mathrm{GeV}$ of the proton off-shell form factors (see the lower and upper solid line, respectively).

In Fig 6 we show auxiliary distribution in $\xi_{1}=\log _{10}\left(p_{1 \perp} / 1 \mathrm{GeV}\right.$ ) (left panel) and $\xi_{2}=$ $\log _{10}\left(p_{2 \perp} / 1 \mathrm{GeV}\right)$ (right panel), where $p_{1 \perp}$ and $p_{2 \perp}$ are outgoing proton transverse momenta. For example $\xi=-1$ means proton transverse momenta $0.1 \mathrm{GeV}$. The biggest contribution for the classical bremsstrahlung process comes from the region $\xi_{i} \approx-0.5$ (i.e, $p_{i \perp} \approx 0.3 \mathrm{GeV}$ ). The distributions in $\xi_{1}$ or $\xi_{2}$ are different because we have limited to the case of $\eta_{\gamma}>0$ only.

In Fig.7 we show corresponding two-dimensional distributions in $\left(\xi_{1}, \xi_{2}\right)$ in a full range of photon (pseudo)rapidity. Quite different pattern can be seen for different mechanisms. For the classical bremsstrahlung we observe an enhancement along the diagonal. This enhancement is a reminiscence of the elastic scattering for which $\xi_{1}=\xi_{2}$. Photon rescattering on the pion cloud (panel c) and photon rescattering with pomeron exchange (panel d) are concentrated at small $\xi_{1}$ or $\xi_{2}$. 

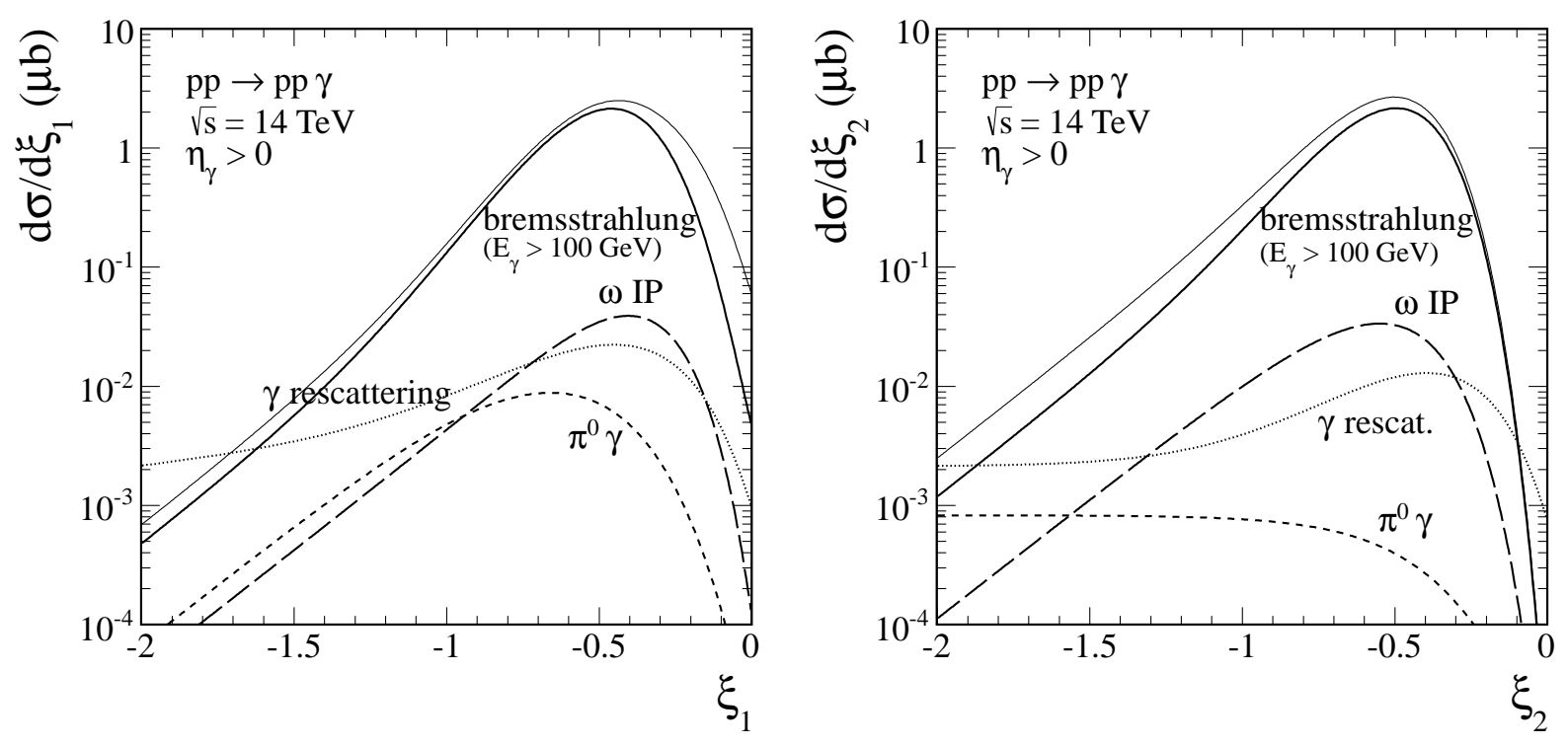

FIG. 6. Distribution in $\xi_{1}=\log _{10}\left(p_{1 \perp} / 1 \mathrm{GeV}\right)$ (left panel) and $\xi_{2}=\log _{10}\left(p_{2 \perp} / 1 \mathrm{GeV}\right)$ (right panel) at $\sqrt{s}=14 \mathrm{TeV}$ and $\eta_{\gamma}>0$. For classical bremsstrahlung $E_{\gamma}>100 \mathrm{GeV}$ and we have used two values of $\Lambda_{N}=0.8,1 \mathrm{GeV}$ (see the lower and upper solid line, respectively) in the proton off-shell form factors.

Photon (pseudo)rapidity distribution is particularly interesting. In Fig 8 we show both distribution for photons $\eta_{\gamma}$ (left panel) and corresponding distribution for outgoing protons $\eta_{p}$ (right panel) for all processes considered in the present paper. In this variable both protons and photons are localized in a similar region of pseudorapidities (or equivalently polar angles). The classical bremsstrahlung clearly gives the largest contribution. It is also concentrated at very large $\eta \gamma$ i.e. in the region where ZDC detectors can be used. We observe a large cancellation between the corresponding terms in the amplitude (2.1) and (2.3) (see left panel) or (2.2) and (2.4). The photon rescattering process clearly dominates in the region of $\eta \gamma$. The cross section for this process is rather small. Clearly an experimental measurement there would be a challenge.

In a first experimental trial one could measure only photons and perform a check for rapidity gap in the midrapidity region. If protons are measured in addition, one could analyze also some new observables related to protons. In Fig 9 we show distribution in the four-momentum transfer squared between initial and final protons. One can observe a change of slope of the $t$ distribution which is caused by the bremsstrahlung of photons. In our simplified model we have assumed a constant (in $t_{1}$ and $t_{2}$ ) energy-dependent slope.

In Fig 10 we show distribution in two-dimensional space $\left(t_{1}, t_{2}\right)$. For the classical bremsstrahlung (left panel) one can observe a ridge when $t_{1} \simeq t_{2}$ which is reminiscence of elastic scattering. The distributions discussed here could in principle be obtained with the TOTEM detector at CMS to supplement the ZDC detector for the measurement of photons.

In Fig 11 we compare distribution in photon-(forward proton) subsystem energy for all processes considered in the present paper. The discussed here $p p \rightarrow p p \gamma$ process gives a sizeable contribution to the low mass $\left(m_{X}>m_{p}\right)$ single diffractive cross section.

If both protons are measured one could also study correlations in the relative azimuthal 

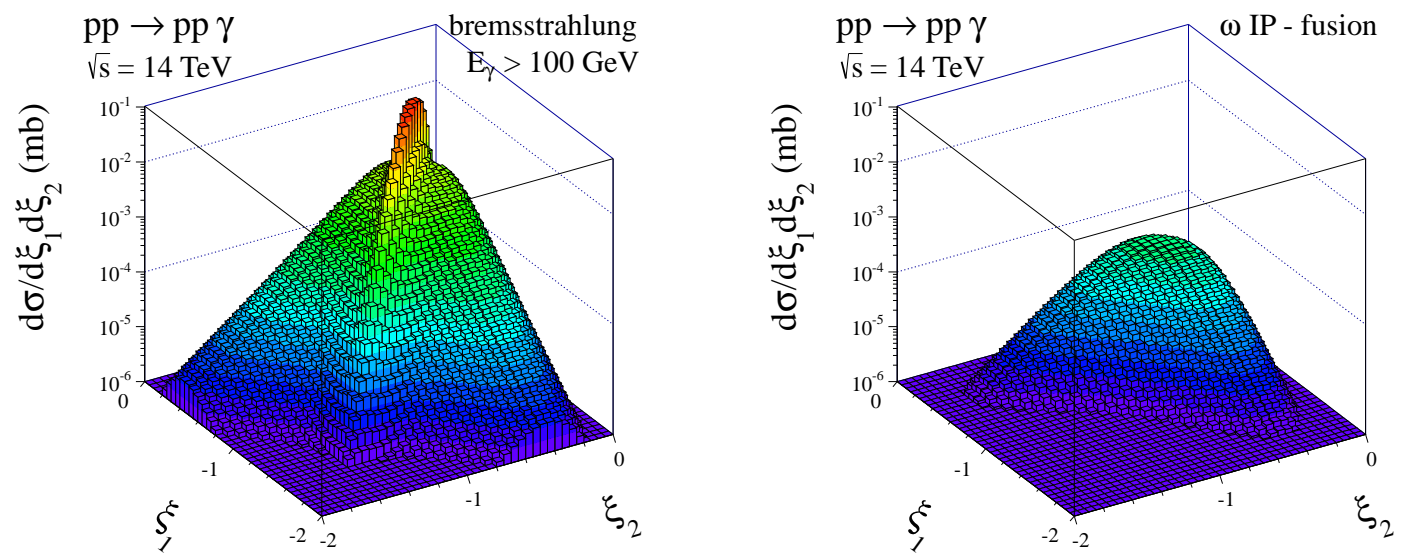

(a)

(b)
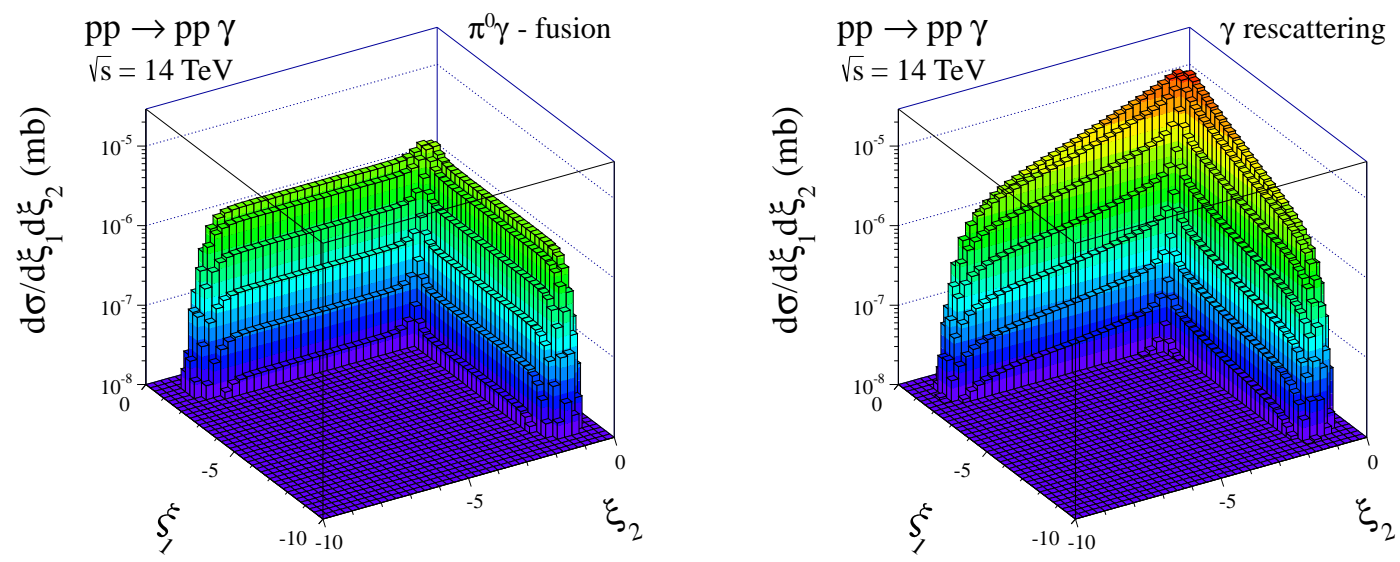

(c)

(d)

FIG. 7. Distribution in $\left(\xi_{1}, \xi_{2}\right)=\left(\log _{10}\left(p_{1 \perp} / 1 \mathrm{GeV}\right), \log _{10}\left(p_{2 \perp} / 1 \mathrm{GeV}\right)\right)$ for the classical bremsstrahlung (a), the omega-rescattering (b), the pion-clould (c) and the photon-rescattering (d) mechanisms at $\sqrt{s}=14 \mathrm{TeV}$. For the classical bremsstrahlung we have imposed in addition $E_{\gamma}>100 \mathrm{GeV}$ and used $\Lambda_{N}=1 \mathrm{GeV}$ in the proton off-shell form factors.

angle between outgoing protons. Our model calculations are shown in Fig.12. One can observe a large enhancement at back-to-back configurations for the classical bremsstrahlung which reminds the elastic scattering case $\left(\phi_{12}=\pi\right)$. The contributions for other mechanisms are significantly smaller and weakly depend on $\phi_{12}$.

\section{CONCLUSIONS}

In this paper we have considered several mechanisms of exclusive single photon production. We have calculated several differential distributions for the $p p \rightarrow p p \gamma$ reaction at high energy for the first time in the literature. The classical bremsstrahlung mechanism turned out to give the biggest cross section concentrated at large photon (pseudo)rapidities. The photons are emitted at only slightly smaller pseudorapidities than the scattered protons. We observe a strong cancellation between the initial and final state radiation. The cross section 

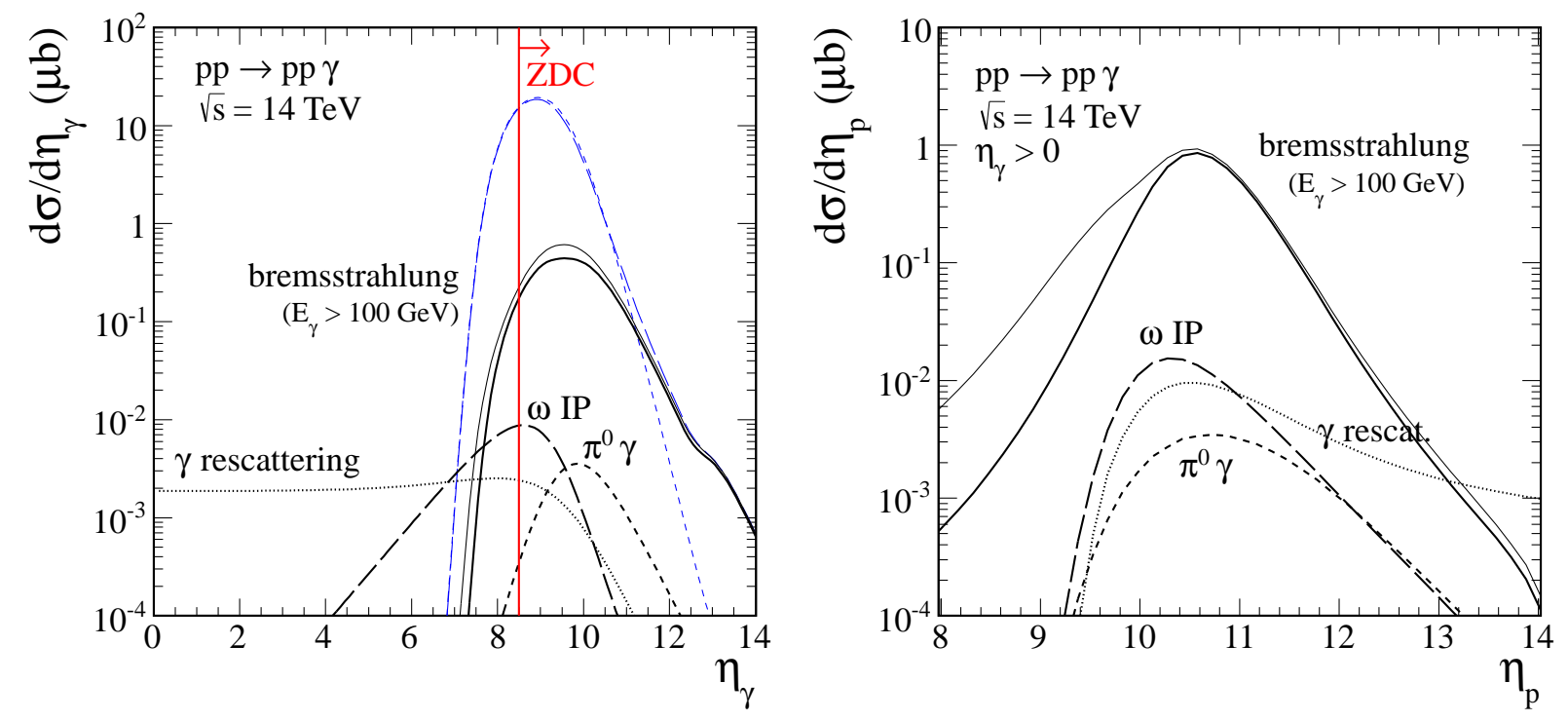

FIG. 8. Distribution in (pseudo)rapidity of emitted photons (left panel) and in pseudorapidity of outgoing protons (right panel) at $\sqrt{s}=14 \mathrm{TeV}$. For classical bremsstrahlung we have imposed $E_{\gamma}>100 \mathrm{GeV}$ and used two values of $\Lambda_{N}=0.8,1 \mathrm{GeV}$ in the proton off-shell form factors (see the lower and upper solid line, respectively). A large cancellation between the initial (2.1) and final state radiation (2.3) is shown (see the blue long-dashed and the blue short-dashed lines, respectively). The lower pseudorapidity limit for the CMS ZDC detector is shown in addition by the vertical line.
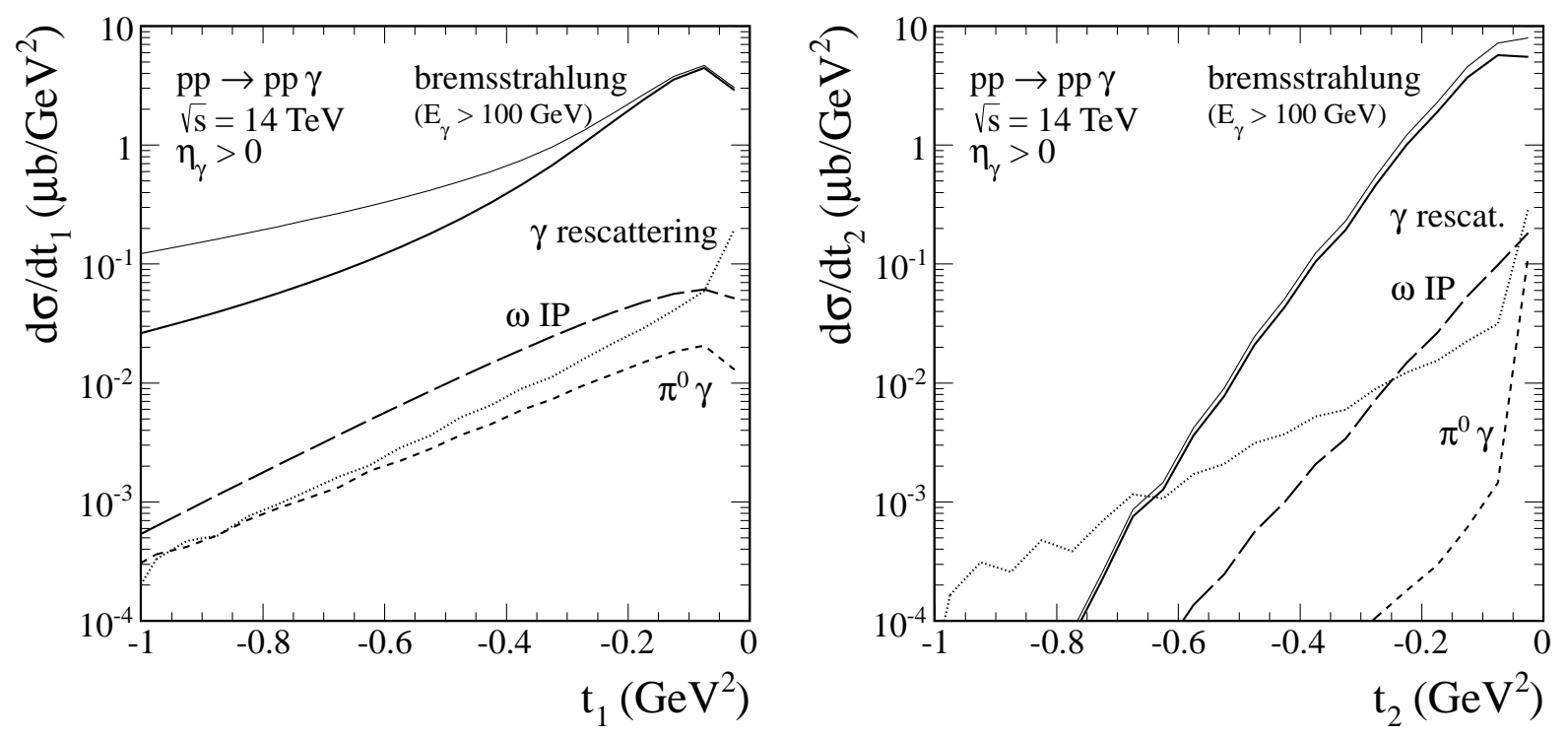

FIG. 9. Distribution in four-momentum squared $t_{1}$ (left panel) and $t_{2}$ (right panel) at $\sqrt{s}=$ $14 \mathrm{TeV}$ and $\eta_{\gamma}>0$. For classical bremsstrahlung we have imposed $E_{\gamma}>100 \mathrm{GeV}$ and used two values of $\Lambda_{N}=0.8,1 \mathrm{GeV}$ in the proton off-shell form factors (see the lower and upper solid line, respectively). 

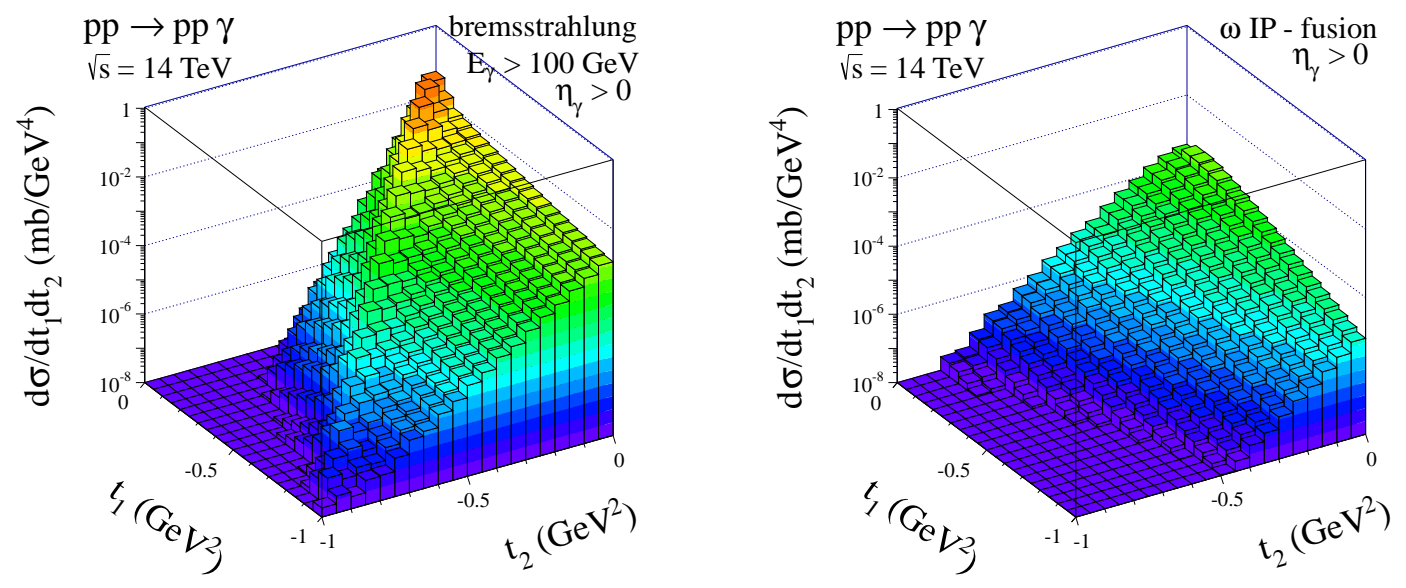

(a)

(b)
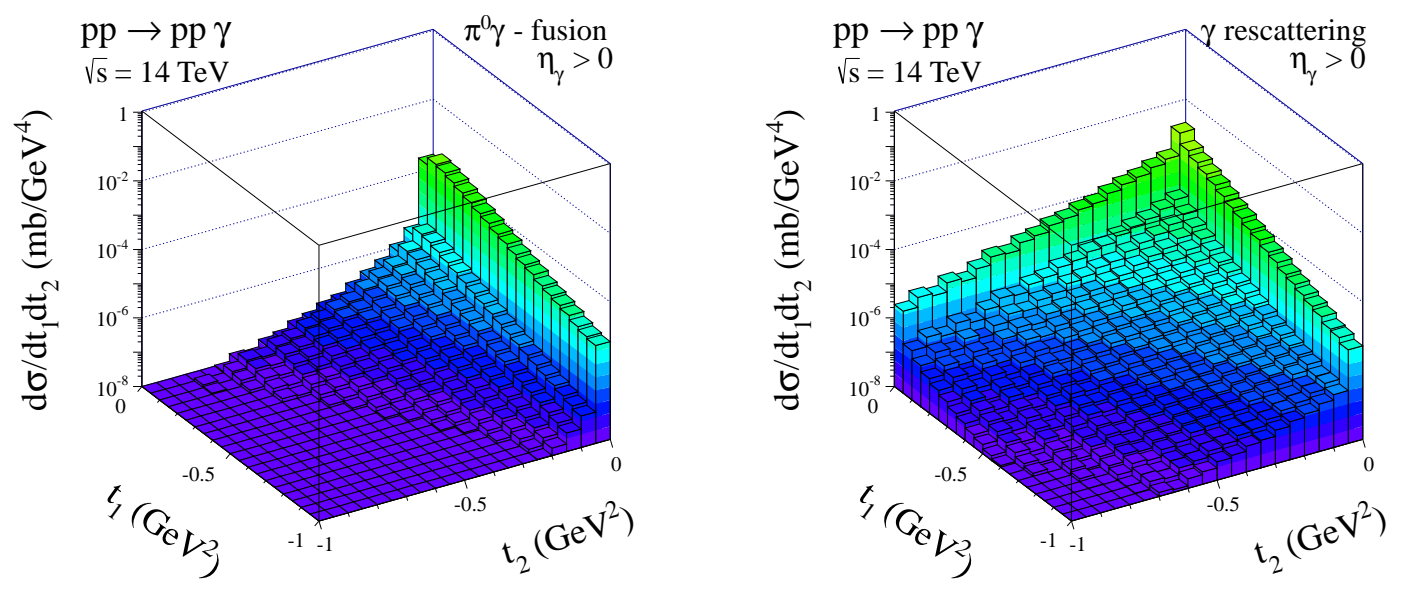

(c)

(d)

FIG. 10. Distribution in $\left(t_{1}, t_{2}\right)$ at $\sqrt{s}=14 \mathrm{TeV}$ and $\eta_{\gamma}>0$ for the classical bremsstrahlung (a) $\left(E_{\gamma}>100 \mathrm{GeV}\right.$ and $\Lambda_{N}=1 \mathrm{GeV}$ ), the omega rescattering (b), the pion cloud (c) and the photon rescattering $(\mathrm{d})$ contributions.

for the classical bremsstrahlung is peaked at back-to-back configurations (similar transverse momenta or polar angles of outgoing protons and relative azimuthal angle concentrated close to $\phi_{12}=\pi$ ). This is a clear reminiscence of elastic scattering. Cut on photon energy $\left(E_{\gamma}>100 \mathrm{GeV}\right)$ reduces the region of $\phi_{12} \cong \pi$ significantly and the integrated diffractive bremsstrahlung cross section is only of the order of $\mu \mathrm{b}$. The cross section for pion-photon or photon-pion exchanges is much smaller. Here both small (photon exchange) and large (pion exchange) four-momentum transfers squared are possible. For this process there is no correlation in azimuthal angle between outgoing protons.

Both classical bremsstrahlung and pion-photon (photon-pion) as well as virtual-omega rescattering mechanisms could be studied with the help of Zero Degree Calorimeters (photons) and the ALFA or TOTEM detectors (protons). By imposing several cuts one could select or enhance the contribution of one of the mechanisms. The cross section for pomeronphoton or photon-pomeron exchanges is rather small and concentrated at midrapidities. Furthermore, the transverse momenta of outgoing photons are small and cannot be easily 


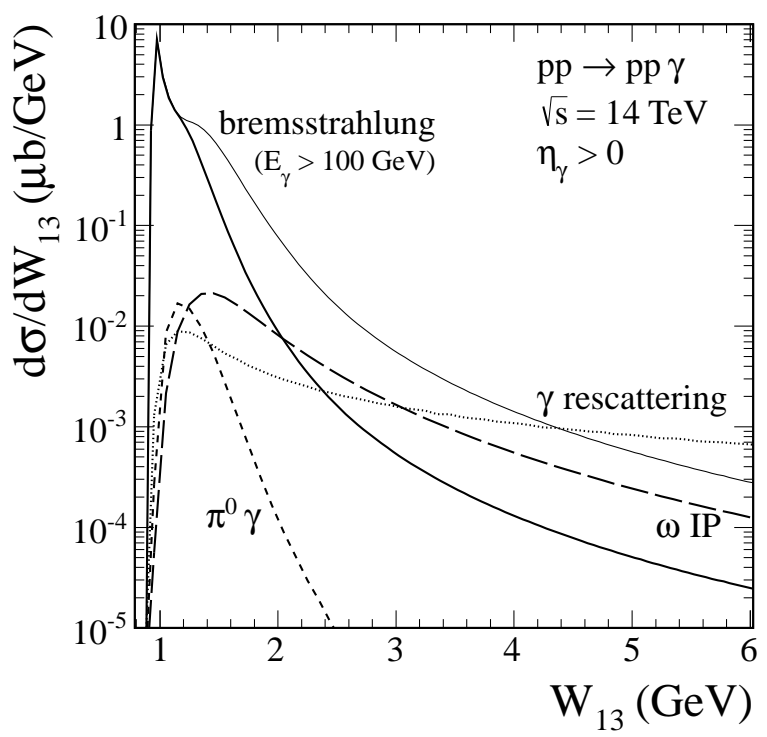

FIG. 11. Distribution in $\gamma p$ subsystem energy $W_{13}$ for all processes considered here at $\sqrt{s}=$ $14 \mathrm{TeV}$ and $\eta_{\gamma}>0$. For classical bremsstrahlung we have imposed $E_{\gamma}>100 \mathrm{GeV}$ and used two values of $\Lambda_{N}=0.8,1 \mathrm{GeV}$ of the proton off-shell form factors (see the lower and upper solid line, respectively).

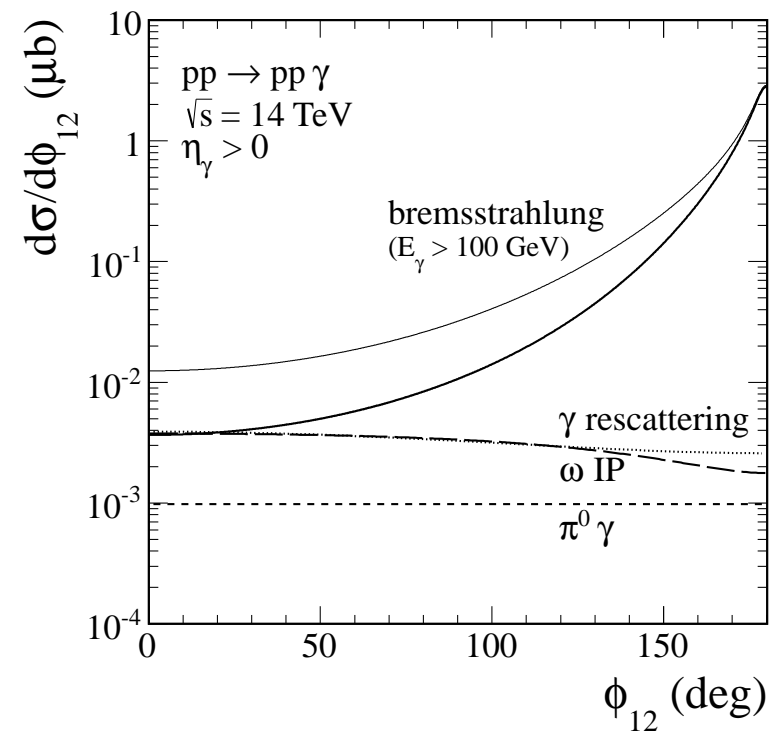

FIG. 12. Distribution in relative azimuthal angle between outgoing protons for different mechanisms at $\sqrt{s}=14 \mathrm{TeV}$ and $\eta_{\gamma}>0$. For classical bremsstrahlung we have imposed $E_{\gamma}>100 \mathrm{GeV}$ and used two values of $\Lambda_{N}=0.8,1 \mathrm{GeV}$ of the proton off-shell form factors (see the lower and upper solid line, respectively).

measured with central ATLAS or CMS detectors.

Summarizing, even present LHC equipment allows to study exclusive production of photons. Since this process was never studied at high energies it is worth to make efforts 
to obtain first experimental cross sections. Since the cross sections are reasonably large one could try to obtain even some differential distributions. This would allow to test our understanding of the diffractive processes and help in pinning down some hadronic and electromagnetic off-shell form factors, difficult to test otherwise.

\section{Acknowledgments}

We are indebted to W. Schäfer for a discussion of some theoretical aspects of our calculation and J. Lämsä, M. Murray and R. Orava for a discussion of some experimental aspects. This work was partially supported by the Polish grant No. PRO-2011/01/N/ST2/04116.

[1] K. Nakayama and H. Haberzettl, Phys. Rev. C80 (2009) 051001;

K. Nakayama and F. Huang, Phys. Rev. C82 (2010) 065201;

A. Johansson, H. Haberzettl, K. Nakayama and C. Wilkin, Phys. Rev. C83 (2011) 054001.

[2] S. Kondratyuk, G. Martinus and O. Scholten, Phys. Lett. B418 (1998) 20;

M.D. Cozma, G.H. Martinus, O. Scholten, R.G.E. Timmermans and J.A. Tjon, Phys. Rev. C65 (2002) 024001.

[3] A. Cisek, P. Lebiedowicz, W. Schäfer and A. Szczurek, Phys. Rev. D83 (2011) 114004.

[4] P. Lebiedowicz and A. Szczurek, a paper in preparation.

[5] S.D. Drell and K. Hiida, Phys. Rev. Lett. 7 (1961) 199;

R.T. Deck, Phys. Rev. Lett. 13 (1964) 169.

[6] G. Alberi and G. Goggi, Phys. Rep. 74 (1981) 1.

[7] V.A. Khoze, J.W. Lämsä, R. Orava and M.G. Ryskin, JINST 6: P01005 (2011), arXiv:1007.3721 [hep-ph];

H. Grönqvist, V.A. Khoze, J.W. Lämsä, M. Murray and R. Orava, arXiv:1011.6141 [hep-ex].

[8] O.A. Grachov et al. (CMS Collaboration), J. Phys. Conf. Ser. 160 (2009) 012059.

[9] J. Peter et al. (ATLAS Collaboration), No. CERN-LHCC-2007-001, No. LHCC-I-016, http://cdsweb.cern.ch/record/1009649.

[10] P. Lebiedowicz and A. Szczurek, Phys. Rev. D83 (2011) 076002.

[11] M. Albrow, A. De Roeck, V. Khoze, J. Lämsä, E. Norbeck, Y. Onel, R. Orava and M.G. Ryskin, arXiv:0811.0120 [hep-ex].

[12] Y. Oh, A.I. Titov and T.-S.H. Lee, Phys. Rev. C63 (2001) 025201.

[13] J. Beringer et al. (Particle Data Group), Phys. Rev. D86 (2012) 010001.

[14] A. Donnachie and P.V. Landshoff, Phys. Lett. B296 (1992) 227.

[15] W. Schäfer and A. Szczurek, Phys. Rev. D76 (2007) 094014.

[16] P. Lebiedowicz and A. Szczurek, Phys. Rev. D81 (2010) 036003;

P. Lebiedowicz, R. Pasechnik and A. Szczurek, Phys. Lett. B701 (2011) 434;

P. Lebiedowicz and A. Szczurek, Phys. Rev. D85 (2012) 014026.

[17] R. Machleidt, K. Holinde and Ch. Elster, Phys. Rep. 149 (1987) 1;

R. Machleidt, Adv. Nucl. Phys. 19 (1989) 189.

[18] N. Kaiser, Phys. Rev. C60 (1999) 057001.

[19] K. Nakayama, Y. Oh, J. Haidenbauer and T.-S. H. Lee, Phys. Lett. B648 (2007) 351.

[20] A. Szczurek and V. Uleshchenko, Eur. Phys. J. C12 (2000) 663.

[21] H. Holtmann, A. Szczurek and J. Speth, Nucl. Phys. A596 (1996) 631.

[22] T.E.O. Ericson, B. Loiseau and A.W. Thomas, Phys. Rev. C66 (2002) 055206. 
[23] S. Donnachie, G. Dosch, P. Landshoff and O. Nachtmann, Pomeron physics and QCD, Cambridge University Press, 2002.

[24] M. Drees and D. Zeppenfeld, Phys. Rev. D39 (1989) 2536. 